\title{
Identification des paramètres d'anisotropie des tôles d'aluminium 1200 et 1050 produites au Cameroun
}

\author{
Bertin Désiré Soh Fotsing ${ }^{1, a}$, Médard Fogué1, Bienvenu Kenmeugne ${ }^{2}$, Guy Fortuné \\ Anago $^{3}$, Brigitte Bacroix ${ }^{4}$, Yves Djimkouo ${ }^{5}$ et Jean-Louis Robert ${ }^{6}$ \\ 1 IUT Fotso Victor de Bandjoun, Université de Dschang, BP 134 Bandjoun, Cameroun \\ 2 IUT de Ngaoundéré, Université de Ngaoundéré, BP 455 Ngaoundéré, Cameroun \\ ${ }^{3}$ Laboratoire d'Études et de Recherche en Technique Industrielles, École Polytechnique d'Abomey-Calavi, \\ BP 2009 Cotonou, Bénin \\ 4 Laboratoire des Propriétés Mécaniques Thermodynamiques des Matériaux, Université Paris 13, Institut Galilée, \\ 99 avenue Jean-Baptiste Clément, 93430 Villetaneuse, France \\ 5 BP 4036 Douala, Cameroun \\ ${ }^{6}$ Laboratoire de Mécanique et Ingénierie, Université Blaise Pascal, BP 2235, Av. Aristide Briand, 03101 Montluçon, France
}

Reçu le 4 mars 2005, accepté le 22 juin 2007

\begin{abstract}
Résumé - L'anisotropie des tôles d'aluminium est induite par le procédé de laminage, elle entraîne des défauts lors de l'opération de mise en forme de pièces par emboutissage. Le travail présenté dans cet article est une étude phénoménologique de cette anisotropie sur des tôles d'aluminium produites à SOCATRAL, au Cameroun. L'objectif est de mettre en évidence l'influence des différents paramètres en vue de mieux contrôler l'évolution de l'anisotropie. Au cours du laminage de l'aluminium, tous les paramètres susceptibles d'avoir une incidence sur la répartition des constituants insolubles dans le métal et sur la déformation des grains du métal ont un effet considérable sur l'anisotropie. Deux nuances d'aluminium (1200 et 1050) ont fait l'objet de cette étude pour laquelle tous les paramètres sont étudiés en utilisant une méthode statistique (SPC) à partir des banques de données de fabrication. Pour chaque paramètre identifié, les courbes de l'évolution de l'anisotropie sont présentées pour différentes épaisseurs de tôle. Les résultats obtenus permettront par la suite d'optimiser l'indice d'anisotropie afin de réduire les défauts à l'emboutissage.
\end{abstract}

Mots clés : Anisotropie / aluminium / laminage / emboutissage / métallurgie

\begin{abstract}
Identification of anisotropy parameters of the sheets of aluminium alloys 1200 and 1050 produced in Cameroon. The anisotropy of aluminium sheets is induced by the rolling process; it involves defects during the operation of parts stamping. The work presented in this article is a phenomenological study of this anisotropy on aluminium sheets produced by SOCATRAL (Cameroon). The objective is to highlight the influence of the various parameters for a better control of the anisotropy evolution. Indeed, during the rolling of aluminium, all the parameters likely to affect the distribution of the insoluble elements in metal and on the deformation of the grains of metal have a considerable effect on the anisotropy. Two aluminium alloys (1200 and 1050) were the subject of this study for which all the parameters are studied by using a statistical method (SPC) from the manufacture data banks. For each identified parameter, the curves of the evolution of the anisotropy are plotted for various sheet thicknesses. The results obtained will thereafter make possible to optimize the index of anisotropy in order to reduce the stamping defects.
\end{abstract}

Key words: Anisotropy / aluminium / rolling process / parts stamping / metallurgy

\footnotetext{
a Auteur pour correspondance :

sohfotsing@ifrance.com
} 


\section{Nomenclature}

\begin{tabular}{|ll|}
\hline ALUCAM : & Aluminium du Cameroun, fonderie d'aluminium \\
$F, G, H, M, N, L:$ & Constantes \\
$H_{i}:$ & Hauteur du godet $i$ en mm \\
$H_{\mathrm{m}}:$ & Hauteur moyenne des godets en mm \\
$I_{\mathrm{s}}:$ & Indice d'anisotropie ou taux de cornes en $\%$ \\
Isomet $:$ & Technique de coulée d'aluminium chez PECHINEY \\
$n:$ & Nombre de cornes sur un godet embouti \\
SOCATRAL $:$ & Société camerounaise de Transformation de l'Aluminium, mise en forme de l'aluminium \\
$\mathrm{S}:$ & Matrice de contraintes \\
$\mathrm{SPC}:$ & Statistical Process Control \\
$T:$ & Température en ${ }^{\circ} \mathrm{C}$ \\
$t:$ & Temps en h \\
$\sigma:$ & Tenseur des contraintes \\
$\sigma_{i j}:$ & Éléments du tenseur des contraintes \\
\hline
\end{tabular}

\section{Introduction}

L'anisotropie des matériaux apparaît aujourd'hui comme une des principales causes de rebut dans les opérations de mise en forme par emboutissage. L'indice d'anisotropie dont l'effet est plus ou moins sensible sur la résistance aux sollicitations, caractérise la variation de la réponse rhéologique du matériau suivant les directions de sollicitations. Cette anisotropie trouve son origine soit dans la structure interne du matériau soit dans les procédés de mise en forme, spécifiquement le laminage dans le cas des tôles. Jusqu'à présent l'anisotropie a toujours été attribuée aux seuls constituants métallurgiques et chimiques du matériau et l'étude de l'anisotropie des matériaux a toujours été faite le plus souvent du point de vue mécanique. Hill [1] développe une théorie générale de modélisation de l'anisotropie plastique des matériaux à partir d'un critère de plasticité écrit sous la forme :

$$
\begin{aligned}
F\left(\sigma_{22}-\sigma_{33}\right)^{2}+G\left(\sigma_{33}-\right. & \left.\sigma_{11}\right)^{2}+H\left(\sigma_{11}-\sigma_{22}\right)^{2} \\
& +2 L \sigma_{23}^{2}+2 M \sigma_{31}^{2}+2 N \sigma_{12}^{2}=1
\end{aligned}
$$

où $F, G, H, M, N$ et $L$ sont des constantes déterminées à partir d'essais établis dans deux directions privilégiées [1].

Fjeldly et al. [2] et Lademo et al. [3] présentent des modèles d'anisotropie des caractéristiques statiques de deux alliages d'aluminium basés sur les critères de Barlat et de Karafillis [2-4]. Dont la fonction d'anisotropie est de la forme $2 \bar{f}^{m}=\alpha_{1}\left|S_{2}-S_{3}\right|^{m}+\alpha_{2}\left|S_{3}-S_{1}\right|^{m}+$ $\alpha_{3}\left|S_{1}-S_{2}\right|^{m}$ où $S_{1}, S_{2}$ et $S_{3}$ sont les valeurs principales de la matrice de contraintes $S=\left\{S_{x} S_{y} S_{z} S_{x y}\right\}^{\mathrm{T}}$ obtenue par une transformation linéaire du tenseur des contraintes; $S=L . \sigma$ avec $\sigma=\left\{\sigma_{x} \sigma_{y} \sigma_{z} \sigma_{x y}\right\}^{\mathrm{T}}$ et $L=\left[\begin{array}{llll}C_{1}+C_{3}-C_{3} & -C_{2} & 0 \\ -C_{3} & C_{3}+C_{1}-C_{1} & 0 \\ -C_{2} & -C_{1} & C_{1}+C_{2} & 0 \\ 0 & 0 & 0 & 3 C_{6}\end{array}\right] \mathrm{m}, C_{1}, C_{2}, C_{3}$ et $C_{6}$ étant des constantes du matériau.

L'objectif de ce travail est de mettre en évidence l'influence des différents paramètres de fabrication des tôles sur l'anisotropie qui est très néfaste lors de l'emboutissage, en vue de mieux contrôler son évolution.
L'indice d'anisotropie est défini par le taux de cornes observés après l'emboutissage, ces cornes sont les irrégularités des bords résultant des déformations plastiques inégalement réparties.

Les nuances d'aluminium 1200 (A4) et 1050 (A5) ont été choisies en raison de leur forte demande sur le marché ( $80 \%$ de la production de SOCATRAL) et pour lesquelles le taux de cornes acceptable doit être inférieur à $6 \%$ pour toutes les épaisseurs.

L'étude commence par la présentation du modèle choisi pour caractériser l'anisotropie, ensuite les différents paramètres induisant l'anisotropie sont étudiés et les différentes courbes d'anisotropie présentées. Une étude critique des différents facteurs d'influence est faite ensuite dans le but d'identifier les facteurs prépondérants afin de proposer par la suite une gamme de fabrication permettant de réduire l'indice d'anisotropie.

\section{Mise en évidence de l'anisotropie}

Une pièce emboutie dans une tôle anisotrope, présente sur son bord, des cornes dont la répartition, le nombre et la hauteur caractérisent le degré d'anisotropie. Pour une tôle parfaitement isotrope, il n'est observé aucune irrégularité du bord. Pour mesurer l'anisotropie des tôles, nous procédons à l'emboutissage de petits godets et nous observons le nombre de cornes apparues, leur hauteur et leur disposition (Fig. 1) [5].

L'indice d'anisotropie des tôles d'aluminium est donné par le taux de cornes qui est le rapport entre la hauteur des cornes observées sur un godet embouti et le nombre de cornes.

Avoir un bon indice revient donc à augmenter le nombre de cornes tout en diminuant leur hauteur.

Pendant l'emboutissage, trois cas de figures peuvent se présenter selon le degré d'anisotropie de la tôle :

1. 4 cornes diamétralement opposées avec deux cornes dans la direction de laminage et les deux autres dans la direction transversale (Fig. 2).

2. 4 cornes diamétralement opposées avec deux cornes orientées à $45^{\circ}$ et les deux autres orientées à $135^{\circ}$ par rapport à la direction de laminage (Fig. 3). 

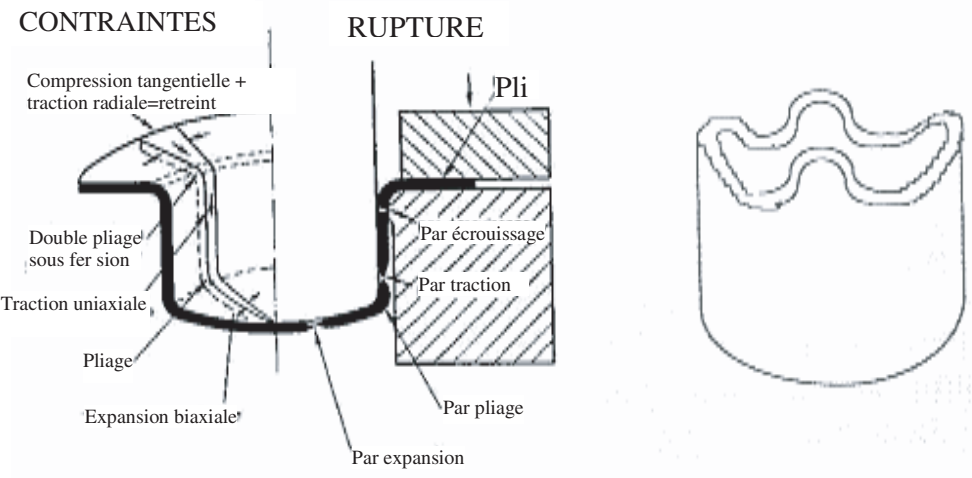

Fig. 1. Emboutissage des godets.

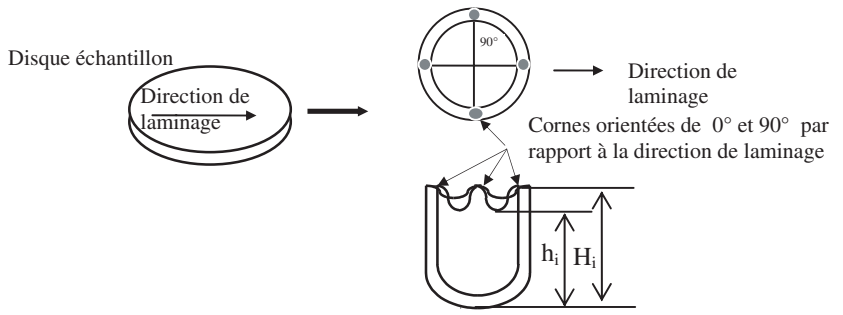

Fig. 2. Cornes à $0^{\circ}$ et $90^{\circ}$.

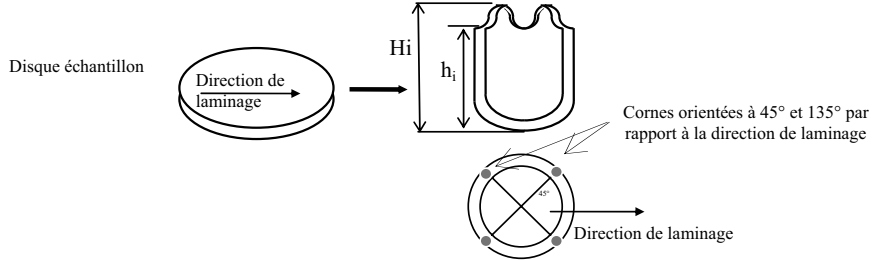

Fig. 3. Cornes à $45^{\circ}$ et $135^{\circ}$.

3. Une combinaison des deux cas précédents; on observe donc la présence de huit cornes (Fig. 4). La présence simultanée de ces deux types diminue les écarts entre la hauteur moyenne des creux et celle des sommets et par conséquent favorise la diminution du taux de cornes.

Ce dernier cas est le plus recherché en fabrication car plus il y a de cornes, moins l'indice d'anisotropie est élevé.

Les types de cornes qui apparaissent le plus sont des cornes orientées à $45^{\circ}$ et à $90^{\circ}$ par rapport à la direction de laminage [5].

L'indice d'anisotropie $I_{\mathrm{s}}$ ou taux de corne est donné par l'expression suivante :

$$
I_{\mathrm{S}}=\frac{\sum_{i=1}^{i=n} H_{i}-\sum_{i=1}^{i=n} h_{i}}{n H_{\mathrm{m}}}
$$

avec

$$
H_{\mathrm{m}}=\frac{\sum_{i=1}^{i=n} H_{i}+\sum_{i=1}^{i=n} h_{i}}{2 n}
$$

où $n$ est le nombre de cornes, $H_{i}$ la hauteur de la corne $i$ et $h_{i}$ la hauteur d'un creux entre cornes (Figs. 2 et 3 ).

\section{Paramètres de l'anisotropie des alliages d'aluminium 1200 et 1050}

Pendant la transformation d'aluminium (coulée des plaques et laminage), tous les paramètres susceptibles d'avoir une incidence sur la répartition des constituants insolubles dans le métal et à la déformation des grains du métal, auront un effet considérable sur l'anisotropie.

Ces paramètres peuvent être regrouper en deux grandes familles, les paramètres métallurgiques et les paramètres de laminage.

\section{- Paramètres métallurgiques}

- mode de coulée des plaques (vitesse de refroidissement),

- format des plaques,

- composition chimique des plaques (teneur en fer et silicium).

\section{- Paramètres de laminage}

- traitement thermique (température de réchauffage des plaques, durée de réchauffage des plaques, recuit intermédiaire),

- les conditions de déformations plastiques (température de début de laminage à chaud, température de fin de laminage à chaud, épaisseur fin de laminage à chaud, taux d'écrouissage).

Pour analyser l'influence de ces différents paramètres sur l'anisotropie du métal produit à SOCATRAL, nous avons constitué deux banques de données pour l'alliage A4 et l'alliage A5 en répertoriant les valeurs de différents paramètres de fabrication pour trois années de production $(1995,1996,1997)$ [6].

Les enregistrements des banques de données sont constitués des paramètres suivants pour chaque opération de laminage :

- $\mathrm{N}^{\circ}$ de plaque

- $\mathrm{N}^{\circ}$ de coulée

$-\%$ fer

- \% silicium

- heure début de réchauffage

- heure fin de réchauffage

- température de réchauffage 


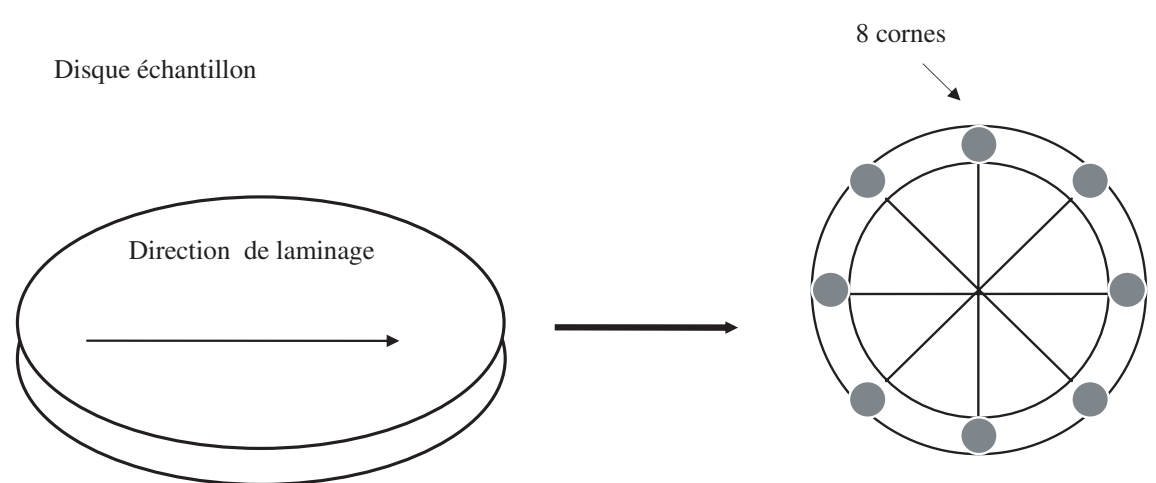

Fig. 4. Combinaison des deux types de cornes.

- température de début laminage à chaud

- température de fin laminage à chaud

- épaisseur ébauche (sortie Duo)

- épaisseur finale

- taux de cornes (valeur moyenne de 2 mesures).

La base de données de l'alliage A5 (BDA5) contient 1894 enregistrements soit 22728 données et celle de l'alliage A4 (BDA4) 1695 enregistrements soit 20340 données.

\subsection{Paramètres métallurgiques}

\subsubsection{Mode de coulée des plaques}

L'anisotropie de l'aluminium est fortement influencée par les conditions de coulée, notamment par la vitesse de refroidissement au cours de la coulée. L'adoption de la coulée à grande vitesse de refroidissement exalte de façon considérable les textures d'orientation du métal recuit [5].

On cherche à combattre ce phénomène, en diminuant la vitesse de refroidissement du métal coulé en continu. C'est le cas de la coulée Isomet couramment utilisée à Issoire (France) où on a cherché à limiter le contact entre le métal solidifié sortant de la lingotière et l'eau de refroidissement. Le métal est refroidi à la sortie de la lingotière à l'aide d'une série de rampes d'eau et d'air comprimé.

Le procédé de coulée Isomet n'est pas réalisé à ALUCAM. Il joue un rôle de régulateur d'isotropie et permet d'obtenir des tôles recuits et isotropes dans une plage d'épaisseur comprise entre 0,6 et $2 \mathrm{~mm}$, alors que le métal obtenu selon le procédé de coulée classique présente des résultats peu favorables.

\subsubsection{Influence de la composition chimique}

Les principaux éléments d'addition utilisés dans la fabrication des alliages 1200 et 1050 sont le fer et le silicium dont les teneurs respectives ne sont pas utilisées directement comme paramètres de laminage. Le paramètre utilisé est le rapport des teneurs fer-silicium.

On peut constater à partir des courbes de la figure 5 que le rapport $\mathrm{Fe} / \mathrm{Si}$ n'a pas un effet considérable sur l'anisotropie du métal transformé à SOCATRAL.

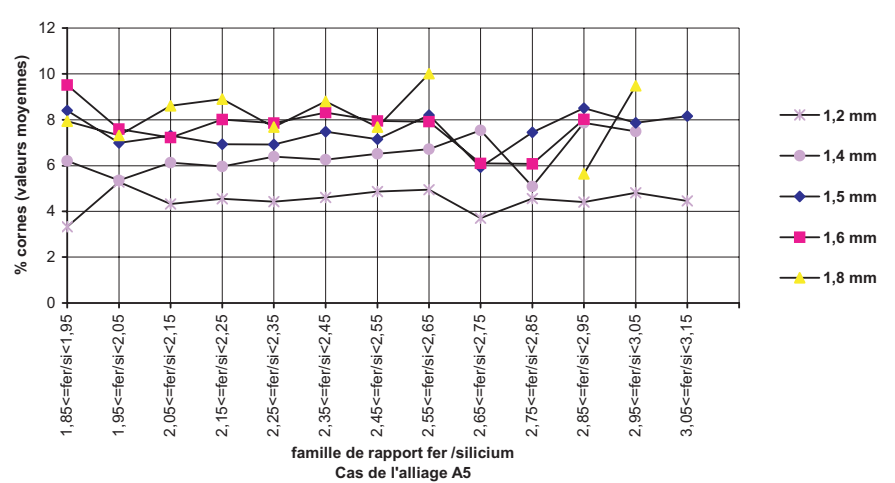

Fig. 5. Taux de cornes en fonction du rapport fer silicium.

\subsection{Paramètres de laminage}

\subsubsection{Influence de la durée de réchauffage}

Le réchauffage ayant pour but de favoriser la malléabilité des plaques lors du laminage à chaud, il joue également un rôle important sur leur homogénéisation. Les figures 6 et 7 montrent l'effet des plaques homogénéisées (durée de réchauffage $>14 \mathrm{~h}$ ) et non homogénéisées (durée réchauffage $<14$ h) sur l'anisotropie de l'A4 et de l'A5.

L'homogénéisation des plaques se fait par un réchauffage à une température d'au moins $540{ }^{\circ} \mathrm{C}$ pendant un temps supérieur à $14 \mathrm{~h}$. On constate que cette opération d'homogénéisation a une influence sur les taux de cornes. Les deux courbes des figures 6 et 7 tout en illustrant l'influence de la durée de réchauffage, montre l'intérêt du traitement d'homogénéisation.

En effet au cours de ce traitement, il y a dissolution des éléments d'addition et nivellement des concentrations de ces éléments dans la matrice (aluminium) [7-9]. On tend vers une homogénéisation de la plaque par une diminution des ségrégations majeures et mineures existantes pour les alliages A4 et A5 transformés par SOCATRAL.

\subsubsection{Influence des durées de réchauffage prolongées}

Les durées de réchauffage prolongées $(t>21 \mathrm{~h})$ améliorent les taux de cornes. La figure 8 présente 


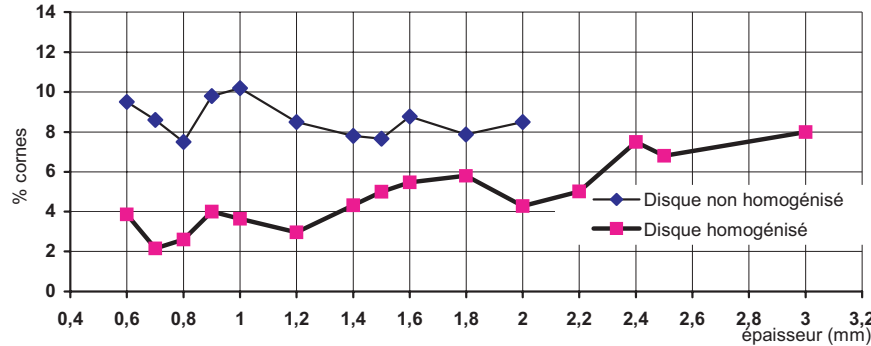

Fig. 6. Taux de cornes en fonction du temps de réchauffage de l'A4.

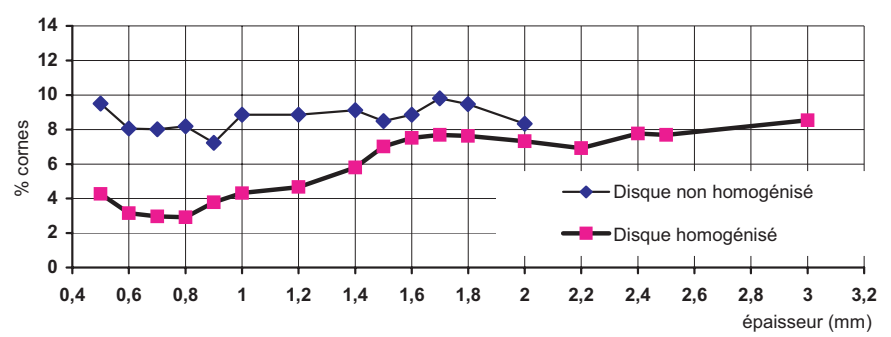

Fig. 7. Taux de cornes en fonction du temps de réchauffage de l'A5.

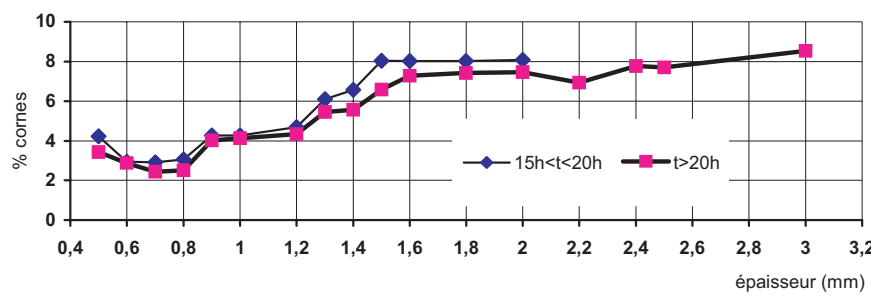

Fig. 8. Influence de la durée de réchauffage prolongée pour l'A5.

l'influence de la durée de réchauffage des plaques destinées à la fabrication des disques isotropes.

La courbe pour une durée de réchauffage comprise entre $15 \mathrm{~h}$ et $20 \mathrm{~h}$ est légèrement au-dessus de celle pour une durée de réchauffage supérieure ou égale à 21 h. Ceci montre qu'après un certain temps de réchauffage et à une certaine température on stabilise : l'effet de la diffusion s'atténue et la plaque est totalement homogénéisée ou a atteint son état d'équilibre.

\subsubsection{Influence de la température de début de laminage à chaud}

La température de début de laminage à chaud est identique à celle de la température de réchauffage puisqu'il n'y a pas un temps d'arrêt entre le défournement et le début du laminage.

Pour observer l'influence de la température de début de laminage, nous avons choisi deux plages de température, $T<550{ }^{\circ} \mathrm{C}$ et $T>550{ }^{\circ} \mathrm{C}$. Pour l'alliage A4, une température de début de laminage supérieure à $550{ }^{\circ} \mathrm{C}$ favorise la diminution de l'indice d'anisotropie pour les épaisseurs comprises entre 0,8 et $1,4 \mathrm{~mm}$. La variation de l'indice d'anisotropie n'est pas

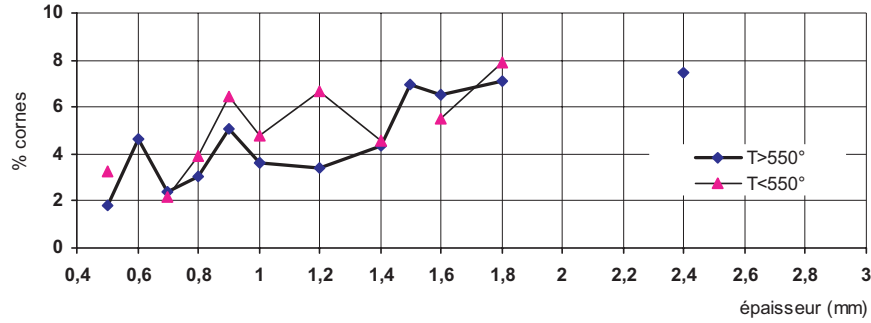

Fig. 9. Influence de la température de début de laminage à chaud pour l'A4.

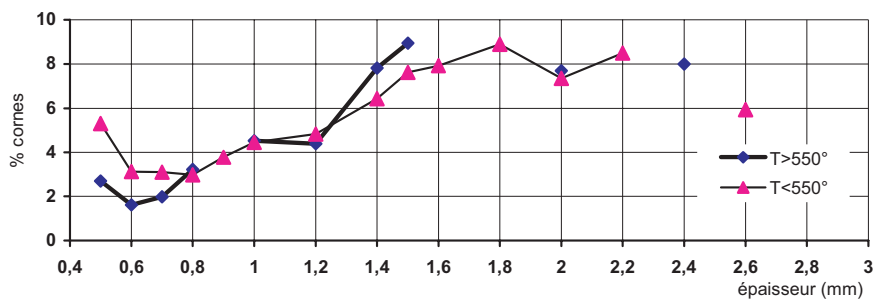

Fig. 10. Influence de la température de début de laminage à chaud pour l'A5.

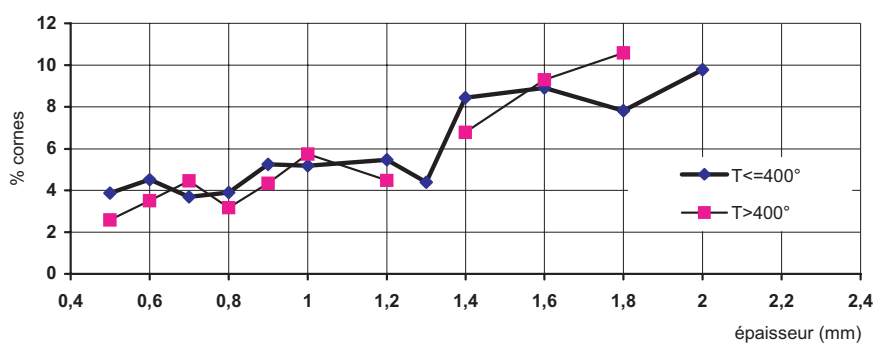

Fig. 11. Influence de la température de fin de laminage à chaud pour l'A4.

significative pour l'A5 dans cette gamme d'épaisseurs (Figs. 9 et 10).

\subsubsection{Influence de la température de fin de laminage à chaud}

Avant le début du laminage, la bande est réchauffée pendant 16 heures, deux plages de température sont utilisées en fonction de l'épaisseur d'ébauche, $T<400{ }^{\circ} \mathrm{C}$ et $T>400{ }^{\circ} \mathrm{C}$. Au cours du laminage à chaud, la température de la bande ne varie pas d'une manière significative. Par conséquent, l'indice d'anisotropie varie très peu (Figs. 11 et 12).

La gamme de laminage est unique au cours du laminage à chaud, et les températures de début de laminage à chaud et de fin de laminage à chaud sont presque identiques pour toutes les plaques, les courbes obtenues sont par conséquent très peu dissociables. Aussi bien pour l'A4 que pour l'A5, on constate que la température de fin de laminage à chaud a très peu d'influence sur l'anisotropie. 


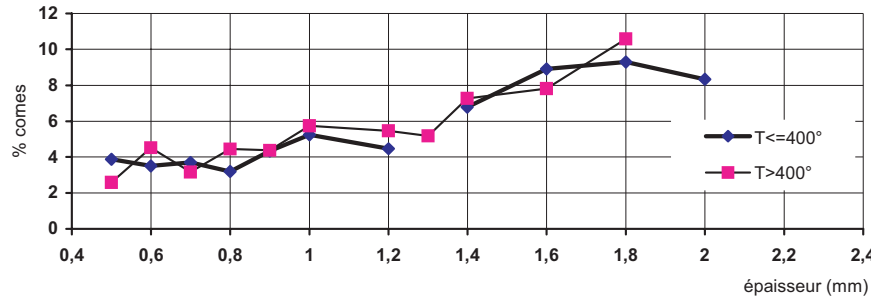

Fig. 12. Influence de la température de fin de laminage à chaud.

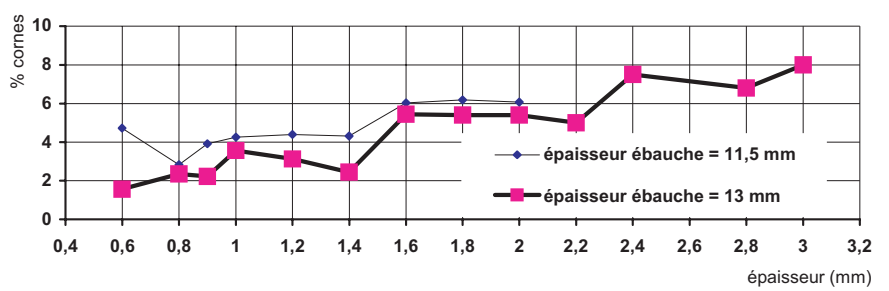

Fig. 13. Influence de l'épaisseur de fin de laminage à chaud (épaisseur ébauche) pour l'A4.

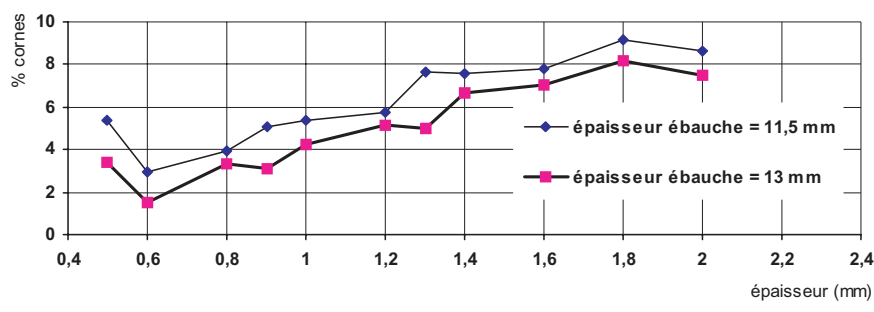

Fig. 14. Influence de l'épaisseur fin laminage à chaud (épaisseur ébauche) pour l'A5 réchauffé pendant 16 h à $540{ }^{\circ} \mathrm{C}$.

\subsubsection{Influence de l'épaisseur de la bande en fin de laminage à chaud}

L'épaisseur de la bande en fin de laminage à chaud influence l'anisotropie, quelque soit l'épaisseur finale du produit. Le laminage à froid d'une ébauche de $13 \mathrm{~mm}$ à une épaisseur finale de 1,3 mm présente un taux d'écrouissage supérieur à celui du laminage d'une ébauche de $11 \mathrm{~mm}$ à une épaisseur finale de 1,4 mm. On constate que plus le taux d'écrouissage est élevé, plus le taux de cornes diminue (Figs. 13 et 14).

D'une manière générale, les figures 6 à 12 montrent que les fortes épaisseurs favorisent l'anisotropie alors les faibles épaisseurs présentent des indices d'anisotropie faibles.

\subsubsection{Influence du recuit intermédiaire}

Le recuit intermédiaire (réchauffage à $540{ }^{\circ} \mathrm{C}$ pendant au moins $16 \mathrm{~h}$ ) est très défavorable. Les plaques ayant subi un recuit précédant le recuit final ont des taux de cornes très élevés.

Les figures 15 et 16 montrent une diminution du taux de cornes de plus de $50 \%$ pour les deux alliages étudiés. Ce fort taux de variation du taux de cornes est une preuve

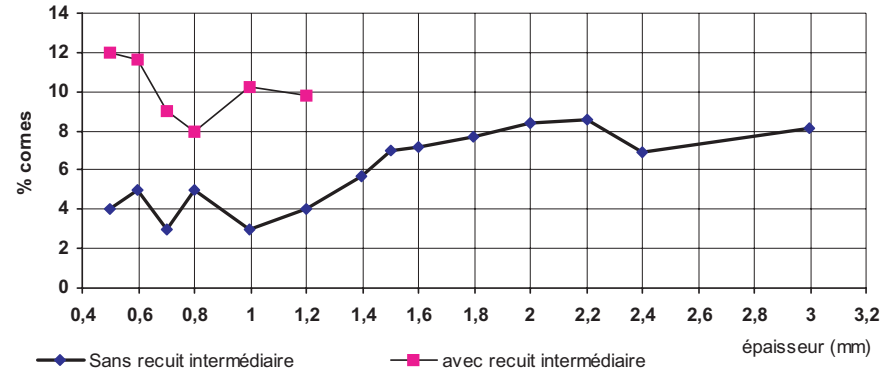

Fig. 15. Influence du recuit intermédiaire pour l'A4.

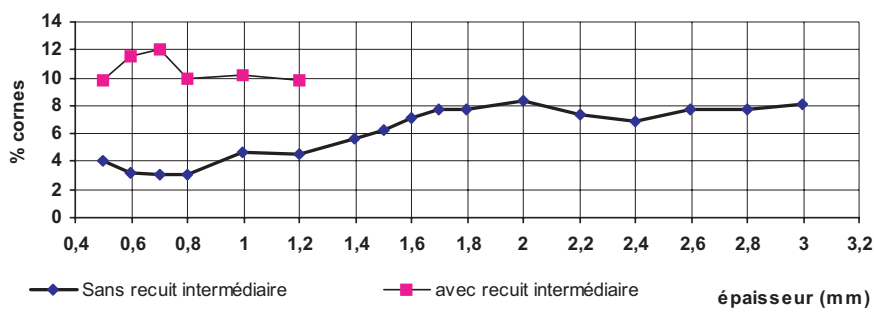

Fig. 16. Influence du recuit intermédiaire pour l'A5.

que le recuit intermédiaire est un paramètre très important de l'anisotropie de l'aluminium laminé.

\subsubsection{Influence de l'écrouissage}

Pour mettre en évidence l'influence de l'écrouissage, nous avons utilisé pour obtenir la même épaisseur finale, des épaisseurs d'ébauche différentes comprises entre $11 \mathrm{~mm}$ et $16 \mathrm{~mm}$. Les courbes des figures 17 et 18 présentent les courbes maximales et minimales obtenues.

On constate dans tous les cas que l'écrouissage est un facteur très important avec des variations du taux de cornes de plus de $100 \%$. Cette variation atteint $800 \%$ pour les épaisseurs de 0,8 mm et 1,6 mm pour l'alliage A4. Pour l'alliage A5 les taux de cornes varient entre $1 \%$ et $13 \%$.

Le taux de cornes diminue lorsque le taux d'écrouissage augmente. Les forts taux d'écrouissage sont obtenus sur les tôles de faibles épaisseurs.

\section{Analyse critique}

Le dépouillement et l'analyse statistique des données de fabrication des disques nous permettent de faire les remarques suivantes :

- Les bandes sorties plus épaisses de la ligne à chaud (épaisseur $>12 \mathrm{~mm}$ ) ont des taux de cornes sur godets peu élevés par rapport aux bandes sorties à des épaisseurs inférieures à $12 \mathrm{~mm}$.

- Les taux de cornes sont inférieurs à $6 \%$ sur les disques de 0,5 à $1,2 \mathrm{~mm}$ d'épaisseur et fortes sur les disques d'épaisseurs supérieurs à 1,2 mm.

- À SOCATRAL, il y a prédominance des paramètres favorisant l'apparition des cornes à $90^{\circ}$ sur ceux favorisant l'apparition des cornes à $45^{\circ}$ (Tab. 1). 


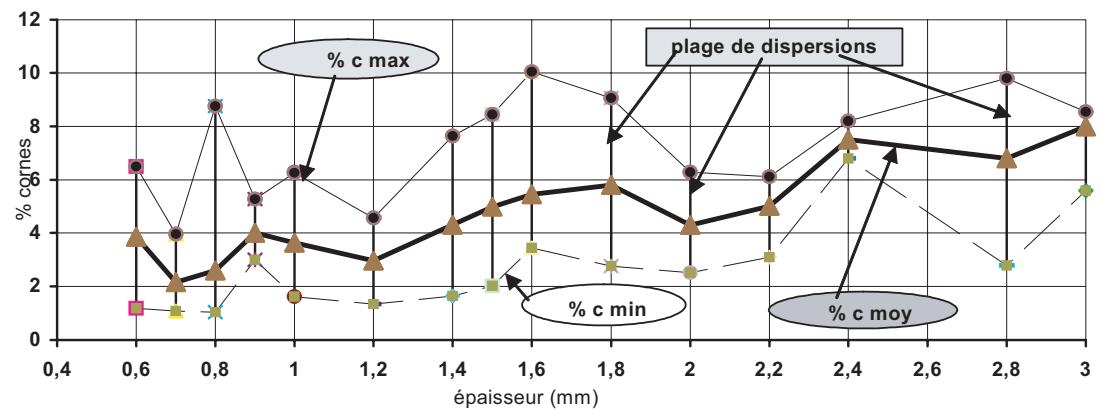

Fig. 17. Influence de l'épaisseur finale pour l'A4.

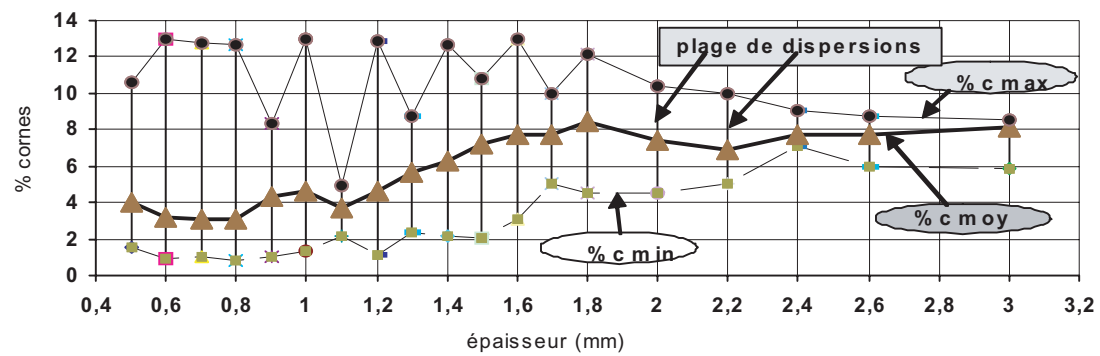

Fig. 18. Influence de l'épaisseur finale pour l'A5.

Tableau 1. Paramètres favorisant l'anisotropie.

\begin{tabular}{|l|l|}
\hline Paramètres favorisant les cornes à $\mathbf{9 0}$ & Paramètres favorisant les cornes à $\mathbf{4 5}^{\circ}$ \\
\hline $\begin{array}{l}\text { Refroidissement rapide de la coulée classique (type } \\
\text { de coulée employée actuellement à la fonderie } \\
\text { ALUCAM) }\end{array}$ & \\
\hline Réchauffage (courte durée de réchauffage) & Réchauffage (longue durée de réchauffage) \\
\hline $\begin{array}{l}\text { Laminage à chaud (température de fin laminage } \\
\text { élevée) }\end{array}$ & Laminage à froid (taux d'écrouissage élevé) \\
\hline Recuit intermédiaire & \\
\hline Teneur élevée en Si & Teneur élevée en fer \\
\hline
\end{tabular}

- L'effet de la température de début et de fin de laminage à chaud est peu observé suite à l'utilisation d'une seule gamme de laminage pendant le laminage à chaud pour les plaques généralement réchauffées à des températures oscillantes autour de $550{ }^{\circ} \mathrm{C}$.

- L'effet de la composition chimique est peu observé. Le rapport fer-silicium intervient très peu sur l'isotropie surtout lorsque l'on désire comme c'est le cas de SOCATRAL se maintenir à des valeurs de taux de cornes inférieures à $6 \%$.

- Le recuit intermédiaire augmente la hauteur des cornes sur godets échantillons. Lorsqu'on récupère une bobine ayant subi un recuit intermédiaire de restauration pour la fabrication des disques qui nécessite un dernier recuit de recristallisation, l'effet du premier recuit se fait immédiatement observé par l'élévation de l'indice d'anisotropie.

Le tableau 1 fait ressortir les différents paramètres qui favorisent l'apparition de l'un ou l'autre type de cornes qui traduisent l'anisotropie du matériau.

\section{Conclusion et perspectives}

Nous avons ainsi procédé à l'identification des différents paramètres qui induisent l'anisotropie des tôles d'aluminium nuances 1200 et 1050. Cette anisotropie est observée par l'apparition de cornes sur des godets d'essais emboutis.

Les taux de cornes acceptables par les emboutisseurs doivent être inférieurs à $6 \%$ pour toutes les épaisseurs de tôles fabriquées, ce taux correspond à l'existence simultanée de cornes à $90^{\circ}$ et de celles à $45^{\circ}$ sur les godets d'essais.

Les paramètres les plus influents sont le taux d'écrouissage, le recuit intermédiaire et le temps de réchauffage.

L'une des perspectives majeures de ce travail est la mise au point de gammes de fabrication qui induisent un très faible indice d'anisotropie $(<6 \%)$ correspondant à une augmentation du nombre de cornes observées sur les godets et une diminution de la hauteur de ces cornes. Il s'agira d'optimiser les différents paramètres pour faire apparaître les cornes à $45^{\circ}$ et à $90^{\circ}$ par rapport à la direction de laminage des tôles. 
Remerciements. Nous tenons à remercier tous ceux qui nous ont permis de réaliser ce travail, notamment le groupe ALUCAM-SOCATRAL qui nous a gracieusement fourni les éprouvettes, et la Coopération Française pour son appui financier.

\section{Références}

[1] R. Hill, Plastic anisotropy and the geometry of yield surfaces in stress space, J. Mech. Ph. Solids 48 (2000) 10931106

[2] A. Fjeldly, H.J. Roven, Phenomenological modelling of the mechanical anisotropy in AlZnMg extruded profiles, Mat. Sc. Eng. A 234-236 (1997) 606-609

[3] O.-G. Lademo, O.S. Hopperstad, K.A. Malo, K.O. Pedersen, Modelling of plastic anisotropy in heat-treated aluminium extrusions, J. Mat. Proc. Tech. 125-126 (2002) $84-88$

[4] A.B. Lopes, F. Barlat, J.J. Gracio, J.F. Ferreira Duarte, E.F. Rauch, Effect of texture and microstructure on strain hardening anisotropy for aluminium deformed in uniaxial tension and simple shear, Int. J. Plast. 19 (2003) 1-22

[5] PECHINEY, Document Technologique de base de Fabrication des bandes et tôles minces non trempées, 1995

[6] Y. Djimkouo, Anisotropie de l'aluminium, Mémoire de DEA en sciences de l'ingénieur, École Polytechnique Yaoundé, 1997

[7] R. Develay, Propriétés de l'aluminium et des alliages corroyés, Techniques de l'Ingénieur, Traité M438, 1992

[8] R. Develay, Traitements thermiques des alliages d'aluminium, Techniques de l'ingénieur, Traité M1290, 1986

[9] H.J. Bunge, C. Esling, Texture et anisotropie des matériaux, Techniques de l'ingénieur, Traité M605, 1997 